\section{Día Mundial de la Salud de 1997: las enfermedades infecciosas emergentes ${ }^{1}$}

Basado en: World Health Organization. World Health Day 1997 Information Kit. Geneva: WHO; 1997. Se publicó en inglés en esta revista, Vol. 1, No. 5, 1997, con el título "World Health Day 1997: emerging infectious diseases".
Hasta hace poco, existía un optimismo generalizado por la creencia de que la lucha contra las enfermedades infecciosas estaba prácticamente ganada. Este optimismo, incitado por ciertos avances, tales como la erradicación de la viruela y el marcado descenso de la incidencia de otras enfermedades, ha generado en algunos casos una actitud de relajamiento que ha permitido que enfermedades como el paludismo y la tuberculosis hayan aparecido nuevamente en muchas partes del mundo. Por añadidura, enfermedades infecciosas que antes no se conocían están apareciendo a un ritmo alarmante: han surgido más de 30 en los últimos 20 años. Simultáneamente, los microorganismos que producen las enfermedades se han vuelto resistentes a una mayor variedad de antibióticos. Todo esto está sucediendo en un momento en que los recursos disponibles para la salud pública están aminorándose en los países ricos y también en los pobres.

En vista del peligro que representan estos factores, la OMS ha elegido el lema de "Enfermedades infecciosas emergentes; alerta mundial, respuesta mundial" para el Día Mundial de la Salud de 1997. Este suceso anual, que se celebra el 7 de abril para conmemorar la adopción oficial de la constitución de la OMS en 1948, dirige la atención hacia un tema de salud de importancia mundial. En su mensaje por el Día Mundial de la Salud, el Director General de la OMS ha expresado el deseo de que el tema de este año sirva de estímulo para que los países examinen objetivamente los problemas relacionados con las enfermedades infecciosas y se concentren en reconstruir desde los cimientos las actividades de vigilancia y control.

\section{LOS PROBLEMAS ACTUALES Y LAS RESPUESTAS FRENTE A ELLOS}

Las enfermedades infecciosas emergentes son causadas por agentes infecciosos de identificación reciente y que no se conocían previamente. Entre ellas figuran una enfermedad respiratoria mortal producida por un hantavirus que apareció en el sudoeste de los Estados Unidos de América en 1993; una variante de la enfermedad de CreutzfeldJakob (afección del sistema nervioso central) que se sospecha, aunque se carece de pruebas, que está asociada con una enfermedad similar del ganado (la encefalopatía espongiforme bovina, o "enferme- 
dad de las vacas locas" en el habla popular); la infección por virus de la inmunodeficiencia humana (VIH), que produce el sida; y la fiebre hemorrágica de Ebola. No siempre se puede saber si estas enfermedades afectan a los humanos por primera vez, o si ya han existido por mucho tiempo sin que se les haya reconocido.

Por otra parte, las enfermedades reemergentes son las que están haciéndose comunes nuevamente, después de haber alcanzado cifras tan bajas que habían dejado de considerarse problemas de salud pública. Estas enfermedades a menudo vuelven a aparecer en proporciones epidémicas. La tuberculosis está aumentando en el mundo entero, debido, en parte, a su asociación con la infección por VIH. El cólera se reintrodujo en 1991 en muchos países de las Américas después de haber estado ausente del hemisferio occidental por varias décadas; su propagación se ha visto facilitada por las deficiencias de los sistemas de abastecimiento de agua y de saneamiento. Ha habido epidemias recurrentes de dengue en zonas urbanas donde el control de los mosquitos se ha deteriorado.

\section{Causas de la emergencia y reemergencia de las enfermedades infecciosas}

Varios factores contribuyen a la aparición y reaparición de las enfermedades infecciosas, pero la mayor parte pueden vincularse con el crecimiento de la población humana; con la frecuencia de los viajes internacionales rápidos; con la sobrepoblación de ciudades con malos sistemas de saneamiento; con cambios en la manera de procesar y manipular grandes cantidades de alimentos; y con la mayor exposición de los seres humanos a los vectores de enfermedades y a sus reservorios naturales. El contacto más estrecho entre el ser humano y los reservorios naturales de un agente infeccioso prepara el terreno para que dicho agente "pegue un salto" en violación de la barrera entre especies, pasando de los animales a los seres humanos.

Estos problemas se ven agravados por el desarrollo y la diseminación de microorganismos resistentes a los antibióticos. Muchos antibióticos bien conocidos ya no son eficaces para el tratamiento de infecciones comunes, como la otitis, la neumonía, la gonorrea y la tuberculosis. Al mismo tiempo, son menos los antibióticos que se lanzan al mercado, en parte como consecuencia del alto costo de desarrollarlos y de conseguir su autorización y del hecho de que la resistencia microbiana reduce su "vida útil." El uso inadecuado de los antibióticos exacerba el problema de la resistencia.

Todos estos factores han sido observados en las últimas décadas, junto con una inquietud decli- nante (y recursos menguantes) en relación con el control de las enfermedades infecciosas. Durante la primera mitad del siglo XX, las defunciones ocasionadas por estas enfermedades se redujeron progresivamente debido al mejoramiento de la higiene y de la nutrición. Esta tendencia fue reforzada por el advenimiento de un número creciente de vacunas y antibióticos. En vista de que las enfermedades infecciosas parecían constituir un peligro cada vez más pequeño, los fondos destinados a su control fueron desplazados hacia la resolución de otros problemas; a medida que se jubilaban los expertos, menos estudiantes entraban a ese campo. La infraestructura del control de las enfermedades infecciosas empezó a derrumbarse y en muchos lugares ha dejado de tener la capacidad para hacer frente a las necesidades que imponen una población en crecimiento y el peligro de nuevas enfermedades.

\section{La respuesta mundial}

La inquietud que han despertado las enfermedades emergentes y reemergentes ha suscitado en los últimos 5 años una serie de iniciativas nacionales e internacionales destinadas a restablecer y mejorar la vigilancia y el control de las enfermedades transmisibles. Los Estados Miembros de la OMS expresaron su inquietud en una resolución de la 48a. Asamblea Mundial de la Salud, celebrada en 1995, que instó a todos los Estados Miembros a fortalecer la vigilancia de las enfermedades infecciosas con objeto de poder detectar rápidamente las enfermedades reemergentes e identificar las de aparición reciente. Ese mismo año la OPS preparó un Plan de Acción Regional para Combatir las Enfermedades Nuevas, Emergentes y Reemergentes en las Américas. Dicho Plan servirá de modelo a la OPS para la ejecución de programas e iniciativas en torno a las infecciones emergentes en el hemisferio occidental.

El éxito de las iniciativas dirigidas a combatir las enfermedades infecciosas depende de la capacidad de obtener información sobre ellas y de la disponibilidad de transmitir esa información en los ámbitos nacional e internacional. A fin de apoyar la resolución de la Asamblea Mundial de la Salud, la OMS estableció la División para la Vigilancia y el Control de las Enfermedades Emergentes y de Otras Enfermedades Transmisibles, cuya misión es fortalecer la competencia en este campo en los ámbitos nacional e internacional a fin de asegurar una respuesta oportuna y efectiva a los problemas de salud emergentes y reemergentes.

Se necesita un consorcio mundial de países, organizaciones no gubernamentales, organismos internacionales e individuos para poder hacer frente a la amenaza de las enfermedades emergen- 
tes y reemergentes mediante la garantía de su rápida detección y contención eficaz. La OMS, siendo una de las partes en esta iniciativa de alcance mundial, está fortaleciendo los sistemas de monitoreo del mundo entero para que integren el sistema de detección general. Ya existen tres sistemas independientes que incluyen laboratorios especializados y sistemas de vigilancia de enfermedades en todos los países, y que proporcionan información electrónicamente a la World Wide Web y a otros medios de comunicación internacionales de tipo electrónico e impreso.

Los Centros Colaboradores de la OMS. Uno de estos sistemas de alcance mundial es la red de Centros Colaboradores de la OMS, que incluye laboratorios especializados e instituciones con experiencia en el diagnóstico y epidemiología de las enfermedades infecciosas. Durante epidemias recientes, ha resultado evidente que el sistema de Centros Colaboradores de la OMS ya no podía asumir por completo la tarea de hacer frente a las necesidades de carácter mundial. Algunos centros, por dar un ejemplo, no se habían mantenido al corriente de los avances tecnológicos y no podían proporcionar el apoyo necesario en materia de diagnóstico para confirmar el origen de los brotes de enfermedad. Otros centros carecían de suficiente experiencia en la epidemiología de algunas de las enfermedades de más reciente aparición. Por añadidura, no hay suficientes Centros Colaboradores en países en desarrollo para asegurar la autosuficiencia de todas las regiones. Por lo tanto la OMS está solicitando a los gobiernos que provean los recursos necesarios para poner al día a los Centros Colaboradores. La OMS también está facilitando el intercambio de información y de reactivos entre los distintos centros, aumentando el número de centros en los países en desarrollo y asegurando que todos los centros estén conectados por la vía electrónica y que tengan un intercambio periódico de información.

El monitoreo de la resistencia a los antimicrobianos. El segundo sistema mundial incluye las redes de la OMS para el monitoreo y la contención de la resistencia a antimicrobianos: la WHONET para el monitoreo de la resistencia general a los antimicrobianos y el programa GASP para el monitoreo de la susceptibilidad del gonococo a los antibióticos. El aumento de la resistencia a los antimicrobianos redunda en mayores costos debido al uso de combinaciones de antibióticos más caras, mayores tasas de hospitalización por infecciones que previamente se podían tratar con facilidad por medios ambulatorios, y el tiempo perdido en términos de asistencia al trabajo o a la escuela hasta el momento de la curación. Por medio de la WHONET y el GASP, la OMS obtiene periódicamente información estandarizada y fidedigna sobre el estado actual de la resistencia a antimicrobianos, ayuda a los países a usar dicha información para formular buenas políticas nacionales de medicamentos, y aplica la información en el entorno internacional para identificar problemas y fomentar la investigación y el desarrollo de antibióticos.

El Reglamento Sanitario Internacional. El tercer sistema está representado por el Reglamento Sanitario Internacional (RSI), que actualmente constituye la única legislación de salud pública internacional que exige la notificación de las enfermedades infecciosas. Solo tres enfermedades están cubiertas por el RSI: el cólera, la peste y la fiebre amarilla. La OMS no tiene ningún mandato legal que le exija hacer obligatoria la notificación y algunos países se muestran renuentes a notificar casos de estas enfermedades debido a las repercusiones negativas que ello podría acarrear, como las restricciones del comercio y del turismo. La OMS actualmente está en proceso de reescribir el RSI a fin de convertirlo en un verdadero sistema de alerta mundial en que se fomente la notificación y se notifiquen todas las enfermedades de importancia mundial.

\section{LOS RETOS Y LAS SOLUCIONES QUE OFRECE EL FUTURO}

Para hacer frente a los retos que plantean las enfermedades transmisibles, la visión del siglo XXI es la de un mundo en continua vigilancia y capaz de lidiar con las enfermedades transmisibles mediante una combinación de los siguientes mecanismos: buenos programas nacionales de vigilancia y control de enfermedades; redes mundiales para monitorear las enfermedades infecciosas y alertar al mundo sobre su presencia y la de otros problemas afines en materia de salud pública; el intercambio rápido de información mediante vínculos electrónicos; y medidas eficaces de preparación y respuesta rápida para frenar las epidemias de importancia internacional.

Entre el mundo de hoy y esta visión del siglo XXI existe una brecha gigantesca. La posibilidad de franquearla depende de la formación de asociaciones con un firme compromiso entre individuos, organizaciones y países, con el apoyo de la OPS, la OMS y otras agencias. El reto y las respuestas internacionales que se necesitan para hacerle frente se detallan a continuación. 


\section{La detección temprana de las epidemias}

En un entorno donde la infraestructura de salud pública es pobre, una enfermedad poco común puede pasar desapercibida hasta que se convierte en un grave peligro para la población y hasta que ya no se puede contener con los recursos nacionales. Los laboratorios de salud pública, dondequiera que existan, a menudo están mal abastecidos o carecen de la capacidad para diagnosticar afecciones comunes y evaluar su impacto en la comunidad.

Se necesita una mejor infraestructura nacional para la vigilancia de rutina de las enfermedades comunes, que incluya el fortalecimiento de los servicios ofrecidos por los laboratorios de salud pública y un plan de adiestramiento dirigido a aumentar el número de personas capaces de llevar las actividades de vigilancia normales a escala nacional. La vigilancia proporciona la información de fondo que hace resaltar los sucesos insólitos para su fácil identificación.

\section{Respuesta nacional rápida a fenómenos patológicos insólitos o a brotes de enfermedad}

Aun cuando se notifica una enfermedad poco común o un brote de enfermedad a las autoridades de salud locales o nacionales, no necesariamente se desencadena una respuesta, o puede que la respuesta sea inadecuada o tardía. Por tal motivo, es necesario adiestrar a personas clave en los países, evaluar los sistemas de vigilancia y preparar planes para contener brotes futuros antes de que se conviertan en emergencias nacionales. Además de estas actividades a largo plazo, las agencias internacionales como la OPS y la OMS, junto con sus socios, tendrán que tomar participación activa en el manejo de los brotes mediante la provisión de asesoramiento por expertos, reactivos diagnósticos, vacunas y medicamentos, así como de un equipo de respuesta internacional en casos necesarios, y mediante la evaluación de la respuesta con miras a mejorar las operaciones futuras.

\section{Sistemas nacionales de vigilancia eficientes y viables}

Muchos países carecen de un sistema nacional $\mathrm{y}$ uniforme de vigilancia para el monitoreo de rutina de las enfermedades transmisibles. Los datos y la información que proporciona un sistema fragmentado y mal integrado no pueden servir para dar la voz de alarma sobre enfermedades ni permiten el monitoreo mundial de las enfermedades transmisi- bles. Tampoco ayudan a las autoridades a formular políticas de salud pública.

Existe la necesidad de establecer pautas para la vigilancia, partiendo de las definiciones de casos aceptadas en el ámbito internacional, y de estimular el uso de dichas pautas por medio de talleres para miembros clave del personal en los niveles regional y nacional. Es preciso facilitar y coordinar el flujo de información entre los diferentes sistemas de vigilancia nacionales que forman parte de una red mundial.

\section{Información oportuna en materia de salud}

Los brotes de enfermedades transmisibles han salido anunciados en las noticias. Los medios de comunicación son, en ocasiones, la única fuente de información sobre los brotes, pero informes inexactos han obstaculizado la evaluación de la verdadera situación y de la necesidad de intervenir. A menudo ha sido difícil obtener información de carácter oficial, o bien porque no existía o porque no se consiguió permiso para diseminarla.

Debe promoverse un intercambio de información abierto y responsable para facilitar la notificación nacional de los brotes de enfermedad. Es necesario poner al alcance de la comunidad mundial información fidedigna y pertinente sobre las enfermedades y sus brotes por medio de los sistemas de comunicación electrónicos y convencionales, suplementados con un asesoramiento adecuado para las personas que viven en las zonas afectadas o que viajan a ellas.

\section{Reacciones acertadas de la comunidad internacional frente a los brotes}

La comunidad internacional en ocasiones ha reaccionado con pánico ante brotes de cólera, fiebre hemorrágica de Ebola y peste y ha impuesto barreras innecesarias contra los viajes y el intercambio comercial, entre ellas la cuarentena en los aeropuertos. Tales medidas producen grandes pérdidas en turismo y exportaciones sin que provean verdadera protección contra la importación de la enfermedad.

Es necesario revisar el Reglamento Sanitario Internacional a fin de ofrecer un código práctico establecido por acuerdo internacional para controlar la propagación internacional de enfermedades infecciosas que encierran un peligro potencial, a la luz de la realidad epidemiológica y económica de hoy. Deben ofrecerse pautas sobre la aplicación del RSI a fin de reducir a un mínimo la interrupción de los viajes y del intercambio comercial que ha ser- 
vido para desalentar otros estados de alerta en ocasiones anteriores.

\section{La infraestructura internacional}

A medida que fueron cambiando las prioridades en el campo de la salud en los años setenta y ochenta, los recursos disponibles para el control de las enfermedades transmisibles escasearon y la infraestructura necesaria se debilitó. La red de Centros Colaboradores de la OMS es un componente importante de esta infraestructura. Hay que fortalecer dichos centros a fin de poder proveer servicios de referencia de calidad para actividades de diagnóstico y adiestramiento y para intervenciones en épocas de brote. También es necesario identificar laboratorios nuevos para extender la red y establecer vínculos electrónicos que faciliten el flujo de información.

\section{La resistencia a agentes antimicrobianos}

La resistencia de bacterias a los antibióticos apareció casi tan pronto se empezaron a usar estos productos y se ha acelerado en las últimas dos décadas. Para hacer frente a este problema, es necesario ampliar el uso de programas que monitorean activamente la frecuencia y distribución geográfica de la resistencia a antimicrobianos. Los usuarios de un programa tendrán que estar conectados a una red de vigilancia internacional para poder generar datos que permitan desarrollar estrategias y pautas mundiales y locales en torno al uso apropiado de los antimicrobianos en seres humanos y animales. Se necesitan investigaciones a fin de mejorar el número de medicamentos disponibles y de idear nuevas formas de prevenir y tratar las infecciones.

\section{La emergencia de enfermedades por contacto con los animales}

La producción intensificada de animales de consumo ha aumentado el peligro de que las enfermedades que afectan a los animales se transmitan al ser humano por medio de la cadena alimentaria. Por otra parte, a medida que se talan los bosques, los animales que habitan en ellos invaden los hábitat más cercanos a las poblaciones humanas y los seres humanos penetran cada vez más en las zonas agrestes restantes por razones de trabajo o de recreo. Estas circunstancias subrayan la necesidad de fortalecer la vigilancia de las enfermedades transmisibles y zoonóticas, de aspirar a un consenso internacional sobre políticas para prevenir y contener la transmisión de enfermedades de los animales a los seres humanos, y de preparar pautas sobre el uso y la manipulación de animales criados para consumo humano.

\section{SYNOPSIS}

\section{World Health Day 1997: Emerging Infectious Diseases}

The World Health Organization (WHO) has chosen the theme "Emerging Infectious Diseases-Global Alert, Global Response" for this year's World Health Day to call attention to the threat posed by infectious diseases that have been recently recognized in humans or that are making a comeback. Factors in the emergence and re-emergence of infectious diseases include deterioration of public health infrastructure, increasing microbial resistance to antibiotics, and social and ecological changes associated with human population growth. WHO emphasizes the need for committed partnerships among countries, international organizations, nongovernmental organizations, and individuals to improve disease surveillance and control capabilities in order to prevent and contain epidemics. For its part, WHO is strengthening the existing three-pronged global monitoring system, consisting of the WHO Collaborating Centers in the area of infectious diseases, the antimicrobial resistance monitoring networks, and the International Health Regulations.

To adequately confront the problem of infectious diseases in the next century, there is a need for strong national disease surveillance and control programs; global disease monitoring and alert networks; rapid information exchange through electronic links, including the World Wide Web; and effective preparedness and rapid response capability to contain epidemics. 